\title{
Reliable Risk Management for Autonomous Vehicles based on Sequential Bayesian Decision Networks and Dynamic Inter-Vehicular Assessment
}

\author{
Dimia Iberraken $^{1,2}$, Lounis Adouane ${ }^{1}$ and Dieumet Denis ${ }^{2}$
}

\begin{abstract}
Guaranteeing the safety of an autonomous vehicle (AV) is a challenging task, especially if the perceived environment is highly uncertain and other road users deviate from their expected trajectories. In this paper, we propose a probabilistic overall strategy for risk assessment and management of $\mathrm{AV}$ in highway through a Sequential Level Bayesian Decision Network (SLBDN) and an appropriate analytical formalization of criteria for anomaly detection based on a Dynamic Predicted InterDistance Profile (DPIDP) between vehicles. Accordingly, the proposed system is designed to take the suitable maneuver decision, have a safety retrospection and verification over the current maneuver risk and take appropriate evasive action autonomously from moving obstacles. Moreover, this probabilistic framework accounts for measurements uncertainty through an Extended Kalman Filter (EKF) and for vehicles' maximum capacities. Since the proposed strategy has a short response time, integrating safety verification in the decision-making process makes real time evasive decisions possible. Several simulation results show the good performance of the overall proposed control architecture, mainly in terms of efficiency to handle probabilistic decision-making even for risky scenarios.
\end{abstract}

\section{INTRODUCTION}

\section{A. Motivation}

One of the major research topics in the domain of autonomous navigation, is enabling vehicles to cope with any environment traffic condition while making the appropriate decision and guaranteeing the safety of maneuvers even in presence of uncertainty [1], [2]. Although multiple Advanced Driver Assistance Systems (ADAS) have successfully improved safety [3], fatal car crashes still occur [4], [5]. This is mainly caused by measurement uncertainties and unexpected maneuvers of other traffic participants. For this reason validating the safety of self-driving vehicles while applying safety verification methods can prove the coherence of the vehicles behavior, reduce remaining risks and the need for extensive testing and more importantly allow us to plan evasive maneuver, in real-time [1], [6], [7].

\section{B. Literature Overview}

Safety in the domain of autonomous vehicles denotes the ability to respect traffic rules and avoid potential collision with other traffic participants. According to [8], the core of a robust automotive safety system able to handle the complexity of driving can be partitioned as a situation assessment

The authors are with ${ }^{1}$ Université Clermont Auvergne, CNRS, SIGMA Clermont, Institut Pascal, F-63000 Clermont-Ferrand, France. First Name. Lastname@uca.fr

${ }^{2}$ Sherpa Engineering, R\&D Department La Garenne Colombe, France. [d.iberraken, d.denis] Csherpa-eng.com method which defines the current driving state of safety while taking into account pre-planned trajectories, and a decisionmaking strategy that makes the control decision. In this paper, both of these parts are developed. Traditional decision making methods involves building a system of rules and deducing the most suitable maneuver [9]. The disadvantages of such systems appear when considering traffic scenarios, unexpected behaviors or perception modules failure that have not been considered during the construction of the system. In this case, new rules need to be added and integrated in the existing system. This increases the complexity of the decision-making process.

Concerning the situation assessment, researchers have pursued multiple ways to improve situation assessment strategies, during vehicle lane change, through threat measure indicators such as Time to Collision (TTC) [10]-[12]. By compromising between optimality and safety, the authors in [13] have introduced a novel interval-based TTC computation, which considers potential uncertainty sources such as vehicular communication latency. However, most works cannot ensure safety if traffic participants deviate from their predefined behavior as most of the classical safety verification approaches, perform the safety assessment offline before the vehicle is deployed.

In order to avoid collision, online safety verification methods have been extensively used in the literature [7], [14], [15]. For example, reachability analysis [16], used as a safety verification method, calculates the reachable set of positions of each vehicle in the environment and possible future collisions are identified when comparing the intersection of the obtained sets. However if the trajectory is regarded as unsafe no alternative is proposed to avoid the collision. Spatial Logic reasoning has also been applied to safety verification, for proving collision freedom of lanechange maneuvers [17] or for real-time spatial logic that can specify constraints in traffic maneuvers on multi-lane motorway [18], however, modeling a generic model using logical formulas is a big challenge.

\section{Contribution}

The proposed paper is to introduce a method to estimate the current performed maneuver risks in real-time from a safety standing point of view for any arbitrary traffic scenarios and investigate the possibility to plan an evasive action (cf. Section II). In previous work [1], [6], we proposed a probabilistic framework which assesses the overall 
surrounding environment by evaluating the collision risk with all observed vehicles, plan driving maneuvers considering predictions of road user trajectories and make the decision on the most suitable actions. This sequencing of decisions is handled by the means of a robust Sequential Level BayesianDecision Network (SLBDN) (cf. Section II) that handles the maneuver decision-making and the safety verification over the current performed maneuver. In this paper, this probabilistic decision-making strategy is extended to handle safety verification (cf. Section II-A) during vehicle lane change and with respect to "all" the vehicles in the environment but also the evasive action selection (cf. Section II-B).

Moreover, using the specific properties of the dynamic predicted inter-distance profile (DPIDP) between vehicles (defined in [6], cf. Section II-A.1), used as risk metric input into the safety verification, anomaly detection criteria are defined (cf. Section II-A.4). In case any anomaly is detected, whether the initial suppositions to perform the maneuver are not anymore confirmed or the perception modules give wrong information on the system, decisions regarding possible evasive maneuver are taken, to ensure the vehicles' safety while accounting for the vehicles' maximum capacities (cf. Section II-B). The Bayesian based approach for handling safety verification and evasive action selection is novel to the best of the authors' knowledge.

In addition, the short-term motion of the ego vehicle and surrounding vehicles are predicted in this paper, based on an Extended Kalman Filter (EKF) (cf. Section II-A.3) which allows to have more reliable AIDP calculation.

This probabilistic decision-making strategy is defined as a part of a Multi-Controller Architecture (MCA) for automated driving in highway (detailed in previous work [1]).

The rest of the paper is organized as follows. Section II formalizes the overall proposed probabilistic decisionmaking process. The simulation results will be presented in Section III and this paper concludes with some prospects on future works.

\section{DeCision-MAKing STRATEgY BASED ON BAYESIAN-DECISION NETWORKS}

It is proposed in this paper a more effective way to take decisions under uncertain conditions, while taking advantage of the dynamic of progression of the inter-distance between vehicles, in order to define better the level of dangerousness of the current maneuver.

The purpose of the overall network is to conform to the driver perception of safety and judgment for dangerous situations and infer the drivers action.

The flowchart presented in Fig. 1, illustrates the sequencing of decisions and the overall safety verification mechanism for all the obstacles present in the environment. The first decision is a part of the Maneuver Decision Level (MDL) where at each $i^{t h} \in \mathbb{N}>N$ sample time, the choice of action regarding the most suitable maneuver is made (cf. Fig.1). The probabilistic decision process is based on the current situation assessment, using the Extended Time To Collision

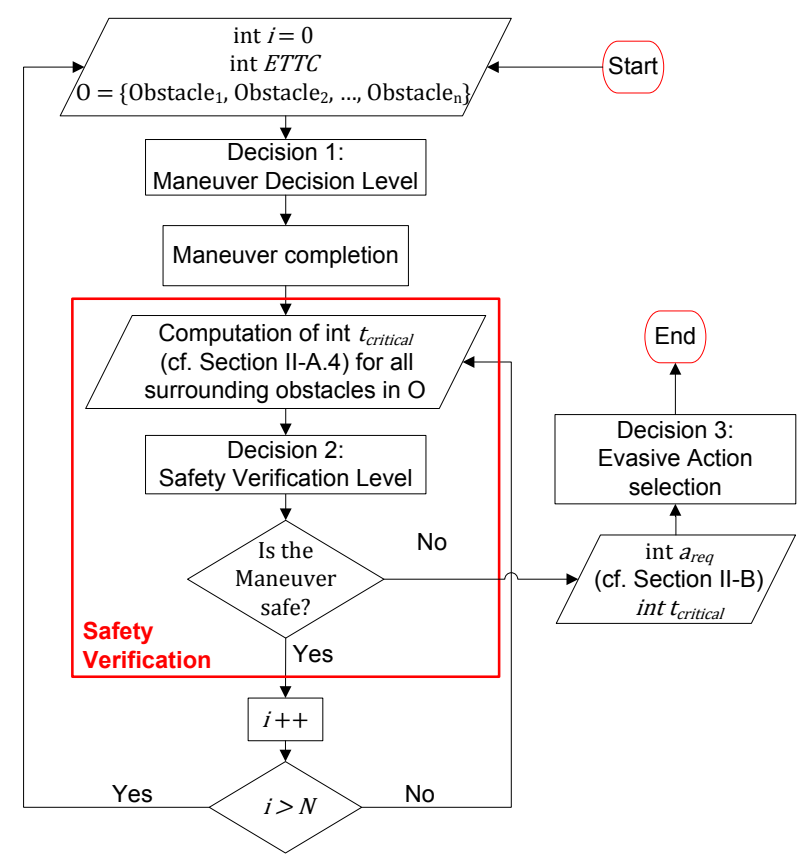

Fig. 1. Flowchart illustrating the sequencing of decisions and safety verifications for all surrounding obstacles. $N$ is an integer value and is defined as $\left[\frac{T_{c h}}{T_{s}}\right]$ with $T_{s}$ the sampling period and $T_{c h}$ is the control observation horizon. $S_{O}$ is the set of visible obstacles in the scene with memory tracking Id. $t_{\text {cirtical }}$ are defined in Section II-A.4. ETTC is the Extended Time To Collision. $a_{r} e q$ is the required acceleration and is defined in section II-B.

(ETTC) [1] while taking measurement uncertainty into account. The possible output maneuvers are: Lane Change Left, Lane Change Right, Keep Lane with ACC, Maintain Velocity with CC.

The second decision is a part of the Safety Verification Decision Level (SVDL) where for each time step $T_{s}$, while the maneuver execution starts, a safety-checking regarding the action chosen in the MDL and a verification of the coherence of the maneuver with the predicted pre-planned trajectory is performed through an improved definition of anomaly detection criteria based-DPIDP [6] (cf. Section IIA), used to detect and compensate for possible failure of the perceptive module or unexpected behaviors.

The third decision is a part of the Evasive Action Decision Level (EADL) (cf. Section II-B) where in case the verification advises to abort the maneuver, the system output the evasive action based on the vehicles maximum capacities and on the endangered lanes.

Since the presented method has a short response time given that Bayesian Networks (BNs) are computationally tractable (due to the exploitation of conditional independence relationships) [9], [19], integrating safety verification in the decision-making process makes real time evasive decisions possible. In addition, Bayes Theory allows uncertainties to be incorporated into calculations and provides a way of combining uncertain data.

A most suitable decision is then obtained by maximizing a utility function over the possible alternatives of the action 
nodes (cf. Section II-C), given the available evidence [19]. We choose discrete actions, instead of low-level controls like steering or accelerating, since modularized systems have been reported to perform better, especially in terms of complexity, functional safety, testability, in autonomous driving than end-to-end systems [20].

\section{A. Safety verification level: Dynamic Predicted Inter- Distance Profile (DPIDP)}

1) DPIDP definition: It is proposed in this paper a safety criteria-based on a DPIDP between vehicles in order to estimate the maneuvers risks during the whole navigation task. Indeed, the assumption considered is that if nothing changes in the initial expected dynamic of all the surrounding dynamic obstacles, the predicted evolution of the interdistance between vehicles is not supposed to change [1].

The DPIDP is built based on predictions of all vehicles future pose. To better understand the approach, we will analyze the system during a lane change maneuver (cf. Fig 2 ), as it is considered among the main risky and challenging maneuvers in highway for autonomous vehicle [21]. An estimation of the time prediction horizon $T_{\text {pred }}[s]$ is then calculated by estimating the required time for the vehicle, given a constant velocity to travel the curvilinear distance of the lane change trajectory.

On the other hand, we suppose that the obstacle-vehicles follow a global path already defined to be the center-line of the lane and the prediction trajectories during the lane change are constructed for $T_{\text {pred }}[s]$ based on their expected behaviors.

For each vehicle pair (ego vehicle and obstacle-vehicle) trajectories, we define a control horizon $N_{c h}$ (number of control moves) to compute the DPIDP as a function of $T_{c h}$ (cf. Eq.(7)). The control time horizon is chosen to be: $T_{\text {ch }}[s]=\max \left(T_{\text {pred }}\right) / M$, where $M$ is a constant value chosen accordingly based on a simple estimation of human reaction time [5].

For each number of control moves $N_{c h}$, the DPIDP will be evaluated between the predicted state vector of the ego vehicle Eq.(1) and the predicted state vector of the chosen obstacle-vehicle Eq.(3) (cf. Fig. 2) and compared to the evolution of the Actual Inter-Distance Profile (AIDP) (cf. Section II-A.3). This gives the system an average time $\left(T_{c h}\right)$ to confirm or not the dangerousness (given by the anomaly criteria in Section II-A.4) of the situation assessment, to act accordingly or to reconfigure otherwise. This way of rea-

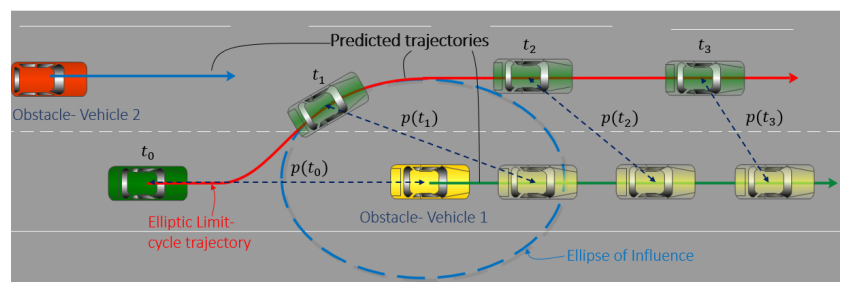

Fig. 2. Predicted Trajectories during lane change maneuver (See. Simulation Video given in https://bit.ly/2G0Zu27)

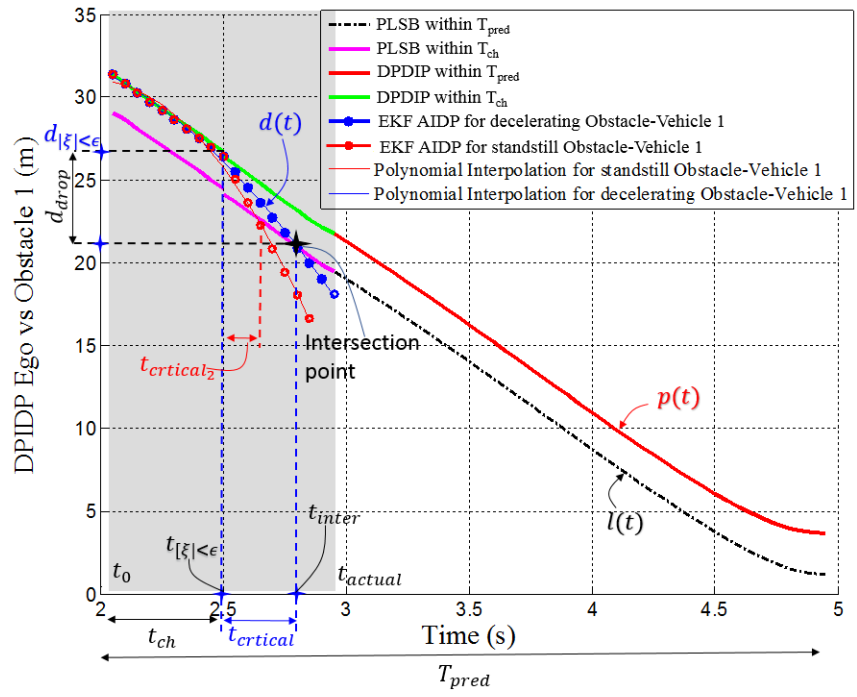

Fig. 3. DPIDP between Ego Vehicle and surrounding Obstacle-Vehicles

soning under uncertainty will eventually help ADAS reduce false alarm and improve performance.

In what follows, the formalisation of the DPIDP is detailed.

2) Analytical formalisation of DPIDP: The motion of the ego vehicle is described by the tricycle model:

$$
\left\{\begin{array}{l}
\dot{x}=v \cos (\theta) \\
\dot{y}=v \sin (\theta) \\
\dot{\theta}=v \tan (\gamma) / l_{b}
\end{array}\right.
$$

Where $X=\{x, y, \theta\}$ is the state vector with $(x, y)$ the vehicle's position and $\theta$ its orientation, $v$ and $\gamma$ are output of the control law (defined in [2]) representing the velocity and the steering angle respectively, $l_{b}$ is the wheel-base of the vehicle.

Based on Euler's Method to solve first order differential equation with a given initial value, we have:

$$
\left\{\begin{array}{l}
x(t+h)=x(t)+h v(t) \cos (\theta(t)) \\
y(t+h)=y(t)+h v(t) \sin (\theta(t)) \\
\theta(t+h)=\theta(t)+h v(t) \tan (\gamma(t)) / l_{b}
\end{array}\right.
$$

with $t \in\left[t_{0}, T_{\text {pred }}\right]$ and $h$ the step size. The motion of the surrounding obstacle-vehicles is assumed to be rectilinear uniformly accelerated and is described by the following equations:

$$
\left\{\begin{array}{l}
x_{o b s}(t+h)=x_{o b s}(t)+\frac{1}{2} a_{x_{o b s}}(t) h^{2}+v_{x_{o b s}} h \\
y_{o b s}(t+h)=y_{o b s}(t)+\frac{1}{2} a_{y_{o b s}}(t) h^{2}+v_{y_{o b s}} h
\end{array}\right.
$$

With $\left(x_{o b s}, y_{o b s}\right)$ the obstacle-vehicle's position, $\left(v_{x_{o b s}}, v_{y_{o b s}}\right)$ the speed components and $\left(a_{x_{o b s}}, a_{y_{o b s}}\right)$ the acceleration components.

The formalization of the DPIDP, defined as function $p(t+$ $h)$ over the interval $t \in\left[t_{0}, T_{\text {pred }}\right]$, is then: 


$$
\begin{aligned}
p(t+h) & =\left(\left(x(t+h)-x_{o b s}(t+h)\right)^{2}+\left(y(t+h)-y_{o b s}(t+h)\right)^{2}\right)^{1 / 2} \\
& =\left(\left(x(t)+h v(t) \cos \left(\theta(t)+h v(t) \frac{\tan (\gamma(t))}{l_{b}}\right)-x_{o b s}(t)-h^{2} \frac{1}{2} a_{x_{o b s}}(t)\right.\right. \\
& \left.-h v_{x_{o b s}}(t)\right)^{2}+\left(y(t)+h v(t) \cos \left(\theta(t)+h v(t) \frac{\tan (\gamma(t))}{l_{b}}\right)-y_{o b s}(t)\right. \\
& \left.\left.-h^{2} \frac{1}{2} a_{y_{o b s}}(t)-h v_{y_{o b s}}(t)\right)^{2}\right)^{1 / 2}
\end{aligned}
$$

A Predicted Lower Safety Boundary $l(t+h)$ is constructed as the projection (parallel curve) of $p(t+h)$ with an offset shift $D_{\text {offset }}$ denoting a possible authorized degree of freedom over the vehicles mutual velocities (cf. Fig. 3) and is defined then as:

$$
l(t+h)=p(t+h)-D_{\text {offset }}
$$

The profiles DPIPD and the PLSB are recalculated each $\left(t_{0}+\right.$ $\left.T_{c h}\right)$.

This above formalisation will allow us to define the anomaly detection criteria (cf. Section II-A.4).

3) Actual Inter-Distance Profile (AIDP) and EKF-based uncertainty handling: The collected sensor data are noisy and uncertain. To deal with these issues, an EKF is used to estimate and predict the ego vehicle and surrounding vehicles state vector from the uncertain sensor measurements. The vehicles motion (Eq.(1)) is described in this paper by the following discretized car-like vehicle evolution model :

$$
\left\{\begin{array}{l}
X_{k+1}=f\left(X_{k}, v_{k}, \gamma_{k}\right)+\varepsilon_{X, k} \\
Y_{k}=g\left(X_{k}\right)+\alpha_{Y, k}
\end{array}\right.
$$

where $\varepsilon_{X, k}$ is zero-mean Gaussian noise representing the process noise and $\alpha_{Y, k}$ is the measurement noise.

Hence, the AIDP is calculated based on the EKF state prediction output and is shown in Figure 4. We can clearly see in the figure that applying the EKF on the noisy AIDP allows to avoid false detections as we will see it in Section II-A.4.

The resulting curve data set: $(t, d(t))$ defined on the interval $t \in\left[t_{0}, t_{\text {actual }}\right]$ is approximated by a polynomial of

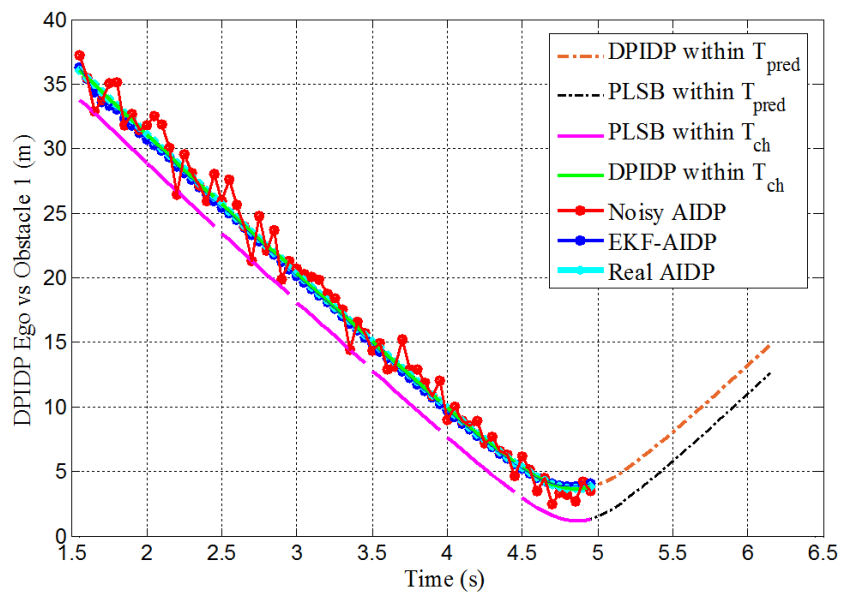

Fig. 4. Filtered AIDP by the means of the proposed EKF second order using the least-squares method that finds the optimal parameter values $\hat{x}=\left(a_{1}, b_{1}, c_{1}\right)$ by minimizing the sum, of squared residuals.

4) Criteria for anomaly detection: Further, contrary to what have been proposed as anomaly criteria in [6], where, at each sample time, is calculated the current available lateral error between the actual inter-distance profile (AIDP) and the DPIDP, it is proposed in this paper to exploit the dynamic of these measures progress to have more pertinent risk assessment and management strategies (cf. Section IIA). We define the anomaly detection criterion as the Critical Time $\left(t_{\text {critical }}\right)$ which is the time interval between the first variation of $d(t)$ and the intersection point between $d(t)$ and the function $l(t)$.

The first variation of the function $d(t)$ is characterized as the time where the error $\xi$ between the evolution of the derivative of the function $d(t)$ with respect to the expected profile $p(t)$ is greater then a small value $\varepsilon$. As for the intersection point $t_{\text {inter }}$ (cf. Fig 3), we have to solve the following quartic equation:

$$
\begin{aligned}
& \left(a_{1}(t+h)^{2}+b_{1}(t+h)+c_{1}\right)^{2}=\left(\left(x+h v \cos \left(\theta-x_{o b s}\right.\right.\right. \\
& \left.\left.+h v \frac{\tan (\gamma)}{l_{b}}\right)-h^{2} \frac{1}{2} a_{x_{o b s}}-h v_{x_{o b s}}\right)^{2}+(y+h v \cos (\theta \\
& \left.\left.+h v \frac{\tan (\gamma)}{l_{b}}\right)-y_{o b s}-h^{2} \frac{1}{2} a_{y_{o b s}}(t)-h v_{y_{o b s}}\right)^{2}-D_{o f f s e t}
\end{aligned}
$$

The positive root value of this quartic equation respecting the condition $t_{\text {inter }}>t_{|\xi|>\varepsilon}$ is the $t_{\text {inter }}$ value.

The critical time will be then:

$$
t_{\text {critical }}=t_{\text {inter }}-t_{|\xi|>\varepsilon}
$$

This criterion combines two information. The first one is that the AIDP crossed the lower boundary (through the calculation of the intersection point). This will allow us to detect the endangered obstacle-vehicles. The second one is the information on criticality of the situation, the smaller $t_{\text {critical }}$ is (due to a quicker deceleration for instance of the ahead obstacle-vehicle to overtake), the steeper the descent is (cf. Fig. 3).

5) Generalisation of the methodology: As a result, for a scene of one ego vehicle and three obstacle-vehicles, the above methodology is applied for each ego-vehicle/obstaclevehicle pair (cf. Fig. 5), resulting thus in three prediction profiles.

\section{6) SVDL nodes:}

- Anomaly detection criteria of AIDP (node AC-AIDP): Depending on the values of $t_{\text {critical }}$, defined in Section II-A.4, input to the node AC-AIDP (cf. Fig. 6), and for any vehicle pair that detects an anomaly, we will have two states:

- Critical time positive means that a value of critical time is found which means $d(t)$ goes beyond the limit safety boundary defined by $l(t)$.

- No Anomaly detected.

The AC-AIDP node constitutes the uncertain observation evidence input to node Status of maneuver $(S M)$. 

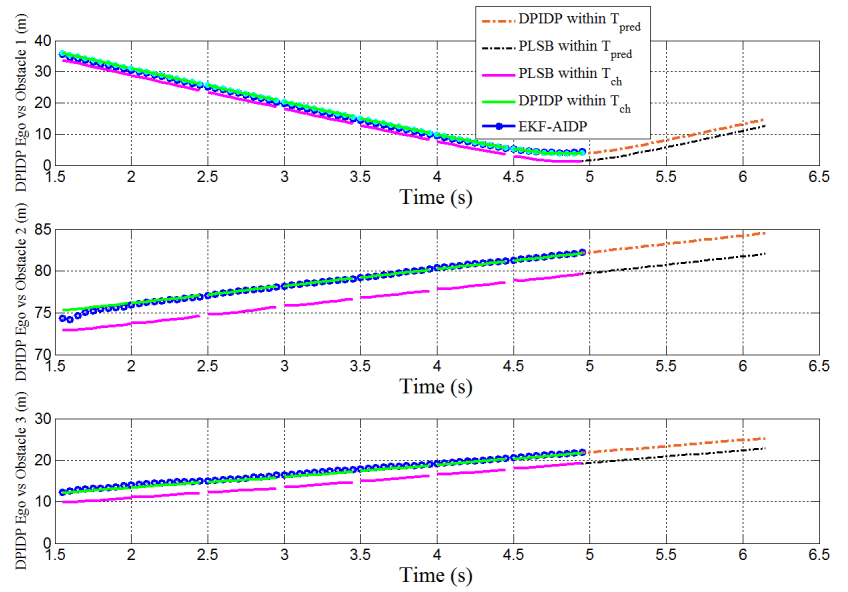

Fig. 5. DPIDP between Ego Vehicle and surrounding Obstacle-Vehicles

- Status of maneuver (node SM): This node describes the status of the engaged maneuver based on the observations that the node AC-AIDP provides. The possible states are Dangerous (for the case where a critical time is found), Safe (the observation AC-AIDP does not endanger the situation).

- Utility Check: $U_{C}$ heck Utility nodes $U_{V}$ defines the cost related to the decision [22]. In the SLBDN (cf. Fig. $6), U_{\text {Check }}$ is the cost related to the safety verification during the lane change maneuver based on the anomaly criteria.

\section{B. Evasive Action Decision Level (EADL)}

In the literature many criteria have been defined [23]-[26] to compute the remaining time span in which the driver can still avoid a collision by braking or by steering. In this paper we propose to compute the required deceleration $a_{r e q}$, based on the definition of the critical time $t_{\text {critical }}$ (cf. Section II-A.4 and Fig. 3) and the evolution of the distance descent (if an anomaly is detected), in order to choose one of the evasive action maneuver. Computing the deceleration will allow us to asses if an emergency braking is possible given the actual situation configuration and given the vehicles' maximum capacity for braking $a_{\max }$. Note that in contrast with previous work [1], [6], where lateral distance errors $\left(E r r_{1}\right.$ between AIDP and DPIDP, $\mathrm{Err}_{2}$ between DPIDP and LSB) is used as anomaly detection criteria, these dynamic aspects and the vehicles' capacities were not taken into account.

Assume that the ego vehicle starts performing a lane change maneuver at an initial speed $v_{0}$ and that a change in the initial configuration happens (obstacle-vehicle 1 comes to standstill for example), meaning that $t_{\text {critical }}$ exists (cf. Fig. 3). Given that we can know the distance drop $d_{d r o p}$ (between the first variation of $d(t): d_{|\xi|>\varepsilon}$ and the intersection point $t_{\text {inter }}$ ) caused by this change and that this distance drop happens during $t_{\text {critical }}$ and assuming a desired stopping inter-distance $d_{\text {stop }}$ (between the ego vehicle and the obstacle-vehicle 1) and a stopping velocity $v_{f}$ at the end of the emergency braking (as the purpose is to avoid the collision), we are able to find the time required $t_{r e q}$ for the ego vehicle to reach $d_{s t o p}$, if $d_{\text {drop }}$ remains constant, as:

$$
t_{\text {req }}=\frac{d_{|\xi|>\varepsilon}-d_{\text {stop }}}{d_{\text {drop }}} t_{\text {critical }}
$$

Starting from the uniform acceleration equation : $v(t)=$ $a t+v_{0}$, we will have the required deceleration to reach $d_{\text {stop }}$ defined by:

$$
a_{r e q}=\frac{v_{f}-v_{0}}{t_{r e q}}
$$

Using the above definitions, we can define the following nodes as input to the EADL:

- Ego Vehicles' Maximum Capacity (MaxCap): Depending on $a_{r e q}$, two states are defined: $a_{r e q} \leq a_{\text {max }}$ and $a_{\text {req }}>a_{\text {max }}$.

- Endangered Lane based Critical Time (E-Lane): Depending on the values of $t_{\text {critical }}$ for each lane and for a road configuration of two lanes (lane information are estimated from OpenSteetMap (OSM) [27] for example), this node has 3 states: Lane 1, Lane 2, Both Lanes on the lanes are endangered and emergency braking is not possible.

- Utility Evasive: $U_{\text {Evasive }}$ is the cost related to the evasive action selection given its input E-Lane and MaxCap.

\section{Decision-Making Strategy}

In the SLBDN network, three decision nodes are represented (cf. Fig. 6). Decision $1\left(D_{1}\right)$ has four possible maneuvers: Lane Change Left (LCL) and Lane Change Right (LCR) for lane change maneuvers, Keep Lane ACC (KLACC) for staying in the considered lane while keeping a safety distance with the vehicle in front and Maintain Velocity (MV) which is an alternative decision allowing to stay in the current lane while maintaining previous velocity configuration. The decision-making in the MDL has been detailed in previous works [1], [6]. Decision $2\left(D_{2}\right)$ has 2 states: Abort Maneuver (AM) that allow us to react to a dangerous change in the DPIDP by canceling the previous decision effect on the system and re-configuring by selecting the appropriate evasive maneuver (cf. SectionII-B) and Maneuver is Safe (MS) state that consolidates the previous decision made in node $D_{1}$ regarding to safety.

Decision $3\left(D_{3}\right)$ in the other side, proposes 3 states for handling anomalies during lane change maneuver (Fig. 2):

1) Continue maneuver $(C M)$ : in case for example only Lane 1 is endangered which means only the pair egovehicle/obstacle-vehicle 1 detects an anomaly $\left(t_{\text {critical }}\right.$ is positive).

2) Emergency braking (EB): in case both lane are endangered which means $t_{\text {critical }}$ is positive for each pair of vehicles in each one of the lane and if the vehicles' maximum capacity for braking allows it $a_{\text {req }} \leq a_{\max }$.

3) Emergency stopping lane (shoulder lane) (ESL): in case both lane are endangered and emergency braking is not possible.

In this paper we show the possibility of handling evasive action selection based on a Bayesian approach during lane 


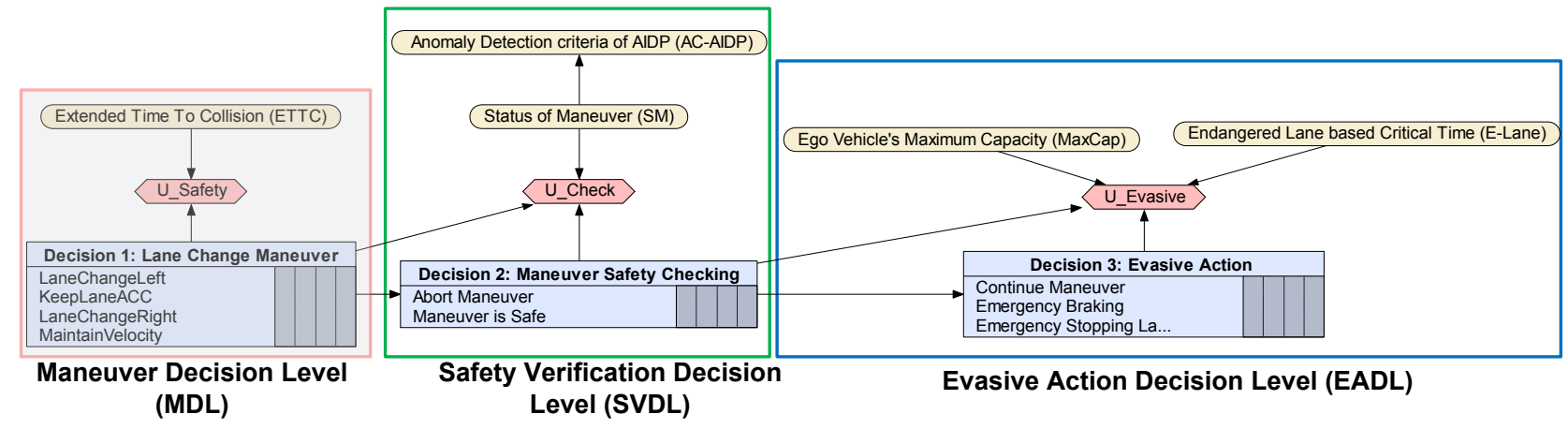

Fig. 6. Sequential Level Bayesian Decision Network (SLBDN) Architecture (developed while using Netica software)

change maneuver that is expandable for the whole navigation.

In order to derive decision strategy, situation assessment variables represented by a set of chance nodes $U_{C}$ has to be defined (cf. Fig. 6). $U_{C}$ represents the set of random variables $\left(X_{1}, X_{2}, . ., X_{n}\right)$ and their conditional probabilistic dependencies [19].

To identify the most suitable decision, we compute the Expected Utility (EU) for each decision state and the final decision is the alternative maximizing this EU. A MultiLevel Decision Network (MLDN) is a representation of a joint expected utility function due to the chain rule:

$$
E U\left(U_{D}\right)=\prod_{X \in U_{C}} P(X \mid \operatorname{parent}(X)) \sum_{w \in U_{V}} U\left(X_{\text {parent }(w)}\right)
$$

The ultimate goal of the proposed cascade decision-making strategy is deriving the most suitable decisions given the available evidence following the temporal order of the set of decision nodes $U_{D}$ (the action chosen for decision $D_{n-1}$ is part of the information available at decision $D_{n}$ ).

\section{Simulation Results}

The simulation results based on experiments performed on a MATLAB/Simulink car simulator has been implemented to test the developed algorithms (cf. Fig.2). To demonstrate the robustness of the proposed approach for handling safe highway maneuvers, let us show in what follows simulation examples.

For the different simulations shown below (See. Simulation Video: https://bit.ly/2G0Zu27), it is considered what follows:

- The scene is constituted of four vehicles in a twolane highway: two vehicles on the right lane including the ego-vehicle (named respectively ego-vehicle and obstacle-vehicle 1) and two vehicles on the left lane (named respectively obstacle-vehicle 2 and obstaclevehicle 3).

- The initial velocities of the vehicles are given by: $V_{\text {ego } \max }=23 \mathrm{~m} / \mathrm{s}, V_{O 1}=12 \mathrm{~m} / \mathrm{s}, V_{O 2}=25 \mathrm{~m} / \mathrm{s} \quad V_{O 3}=$ $5 \mathrm{~m} / \mathrm{s}$.

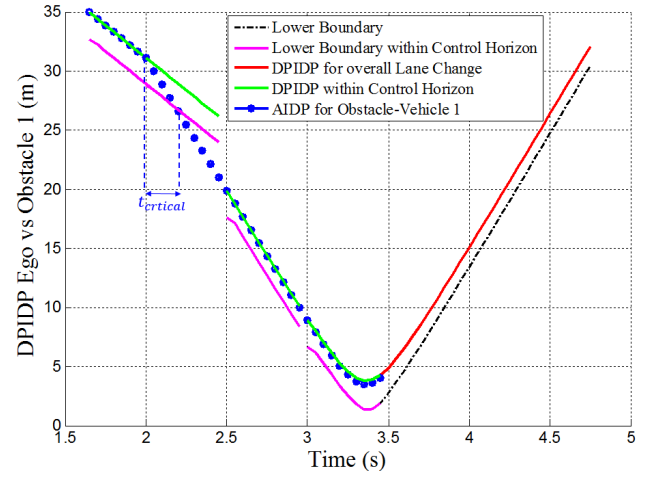

(a) Reconfiguration of the DPIDP

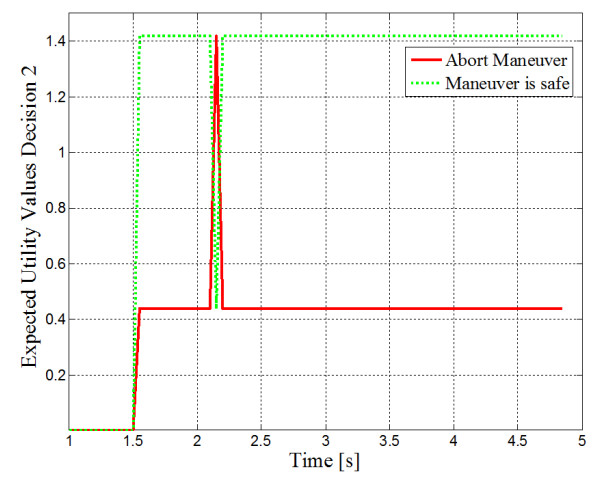

(b) Decision 2: SVDL

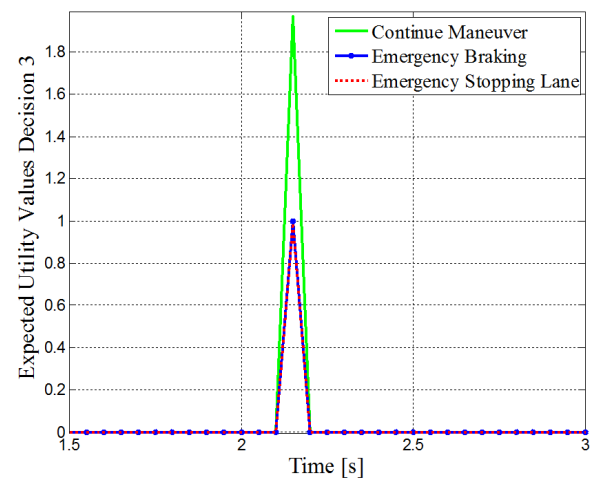

(c) Decision 3: EADL

Fig. 7. DPIDP, Decision 2 and Decision 3 when only lane 1 is endangered 
A. Safety Verification and evasive action: Lane 1 is endangered

We have selected a dangerous scenario that can occur in a highway environment where the obstacle-vehicle 1 in front suddenly brake, while the ego vehicle is trying to perform a lane change maneuver. In this case, we can see in Fig. 7(a) that the AIDP crosses the PLSB generating consequently the SVDL to advise aborting the maneuver (cf. Fig. 7(b)) given that $t_{\text {critical }}$ is positive. In this case, given that the left lane is free and given the ability of the system to reconfigure and adapt to the change (thanks to the properties of the ELC [6] and to the DPIDP) the evasive action maneuver is to continue the lane change maneuver (cf. Fig 7(c)) followed by a reconfiguration of the DPIDP to the new setting which leads to the state ManeuverisSafe for $D_{2}$.

\section{B. Safety Verification and evasive action: Lane 1 and Lane 2 are endangered}

In addition to the obstacle-vehicle 1 that comes to standstill, it appears in the scene the obstacle-vehicle 3 coming from behind in the left lane accelerating. At the beginning of the simulation this obstacle is far and slow enough to allow the lane change maneuver to start but suddenly accelerates. Consequently, two of the three DPIDP profiles (cf. Fig. 8(a)) (corresponding to obstacle-vehicle 1 and obstacle-vehicle 3) alert us through the anomaly criteria that the current situation

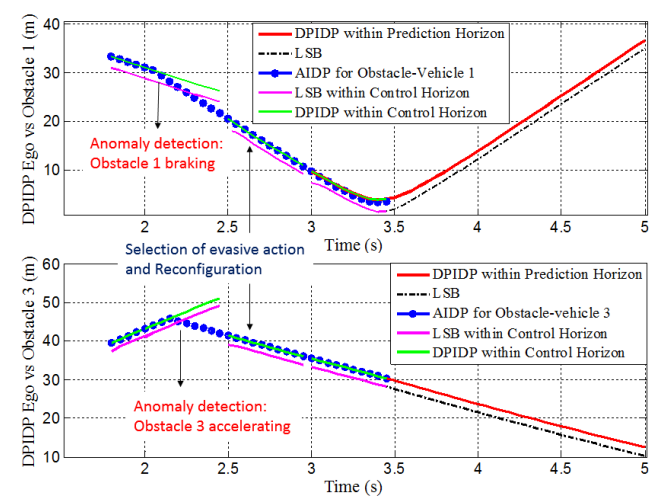

(a) DPIDP for the two obstacles

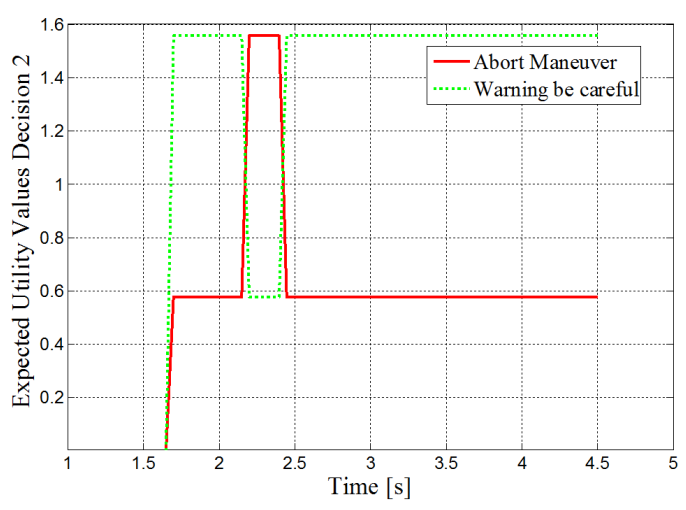

(b) Decision 2: SVDL

Fig. 8. DPIDP and output Decision 2 when both lane are endangered

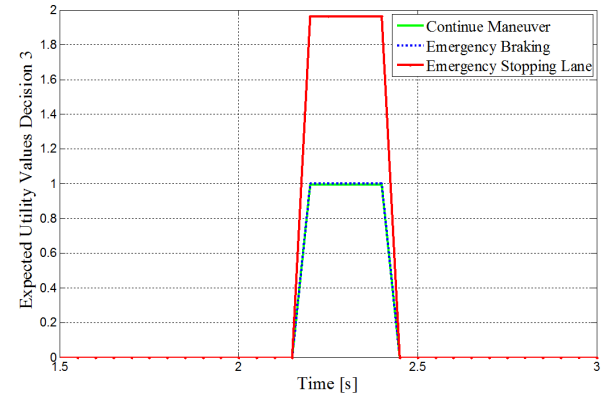

(a) Decision 3: Scenario 2

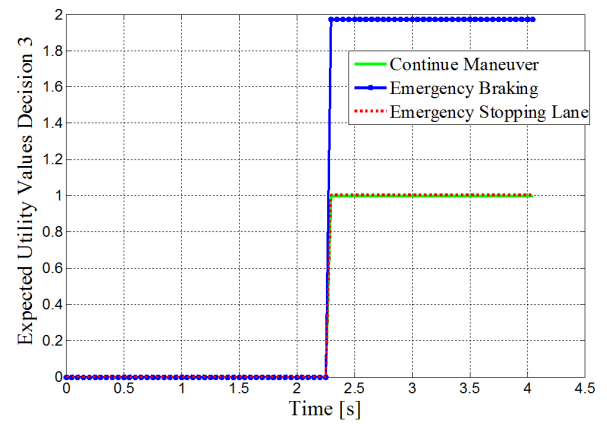

(b) Decision 3: Scenario 3

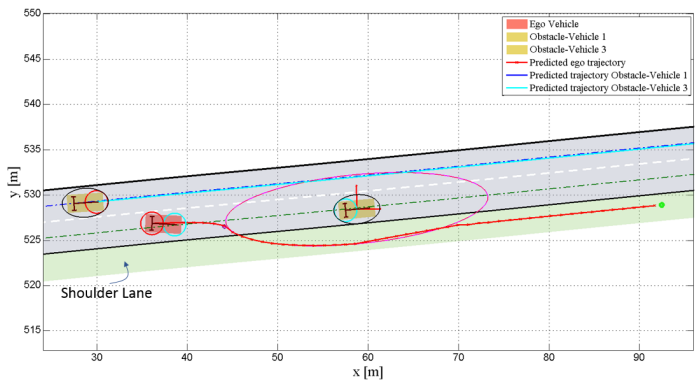

(c) Evading to shoulder lane while using ELC [1]

Fig. 9. Evasive Decisions when both lane are endangered

is dangerous and the lane change maneuver is impossible according to the acceleration capacities of the ego-vehicle.

In this case the appropriate decision is to abort the maneuver (cf. Fig 8(a)) and two different evasive maneuver are possible: Emergency braking or Emergency Stopping Lane. The Emergency braking is possible if and only if $a_{r e q} \leq a_{\max }$. In contrast with previous work [6] where a similar scenario has been shown, the ego-vehicle will automatically try to brake while not accounting for the vehicles' capacity for emergency braking which is not the optimal way. In this second scenario (Scenario 2), $a_{\text {req }}>a_{\max }=-10 \mathrm{~m} / \mathrm{s}^{2}$, which leads the system to choose the Emergency Stopping Lane as evasive action (cf. Fig. 9(c) and Fig. 9(a)). Due to the ability of the system anomaly metric DPIDP to reconfigure within an average control horizon time $T_{c h}$ and adapt to the changes as the safety is ensured, $D_{2}$ come back to status Maneuver is safe.

In a third scenario (Scenario 3), where the deceleration of the Obstacle-vehicle 1 is smoother, $a_{\text {req }} \leq a_{\max }=-10 \mathrm{~m} / \mathrm{s}^{2}$. This induce the third decision to be Emergency braking as 
we can see in Figure 9(b).

\section{CONCLUSIONS}

This paper proposes to enhance the probabilistic overall strategy for risk assessment and management of AV in highway, highlighted in [1] and [6]. The aim of this paper is to ensure even more AV safety in uncertain environment and changing dynamic/behaviors of the surrounding vehicles, while using mainly a Sequential Level Bayesian Decision Network (SLBDN), and an appropriate analytic formulation of anomaly detection criteria based on a Dynamic Predicted Inter-Distance Profile (DPIDP) between vehicles called the critical time $t_{\text {critical }}$, allowing us to quantify the risks and the criticality of the driving situation. In addition, to account for uncertainties in the state vector of the vehicles, an Extended Kalman Filter (EKF) is utilized. The proposed methodology is thus designed to integrate the safety verification algorithms and the evasive action selection into the decision-making process while accounting for uncertainties. This enables us to have a retrospection over the current performed maneuver risks and take the appropriate evasive action maneuver, in real time while accounting for the vehicles' maximum capacities.

Several simulation results show the good performance of the overall proposed control architecture, mainly in terms of efficiency to handle probabilistic decision-making even for risky scenarios. Topics for future work include to further analyze and evaluate the generality the overall proposed approach. Real-time experimentation will also be carried out mainly in collaboration with the R\&D Department of Sherpa Engineering.

\section{ACKNOWLEDGMENT}

This work has been sponsored by Sherpa Engineering and ANRT (Conventions Industrielles de Formation par la Recherche). It is also supported by the French government research program "Investissements d'Avenir" through the RobotEx Equipment of Excellence (ANR-10-EQPX-44) and the IMobS3 Laboratory of Excellence (ANR-10-LABX-1601).

\section{REFERENCES}

[1] D. Iberraken, L. Adouane, and D. Denis, "Multi-level bayesian decision-making for safe and flexible autonomous navigation in highway environment," in 2018 IEEE/RSJ International Conference on Intelligent Robots and Systems (IROS), pp. 3984-3990, Oct 2018.

[2] L. Adouane, Autonomous Vehicle Navigation: From Behavioral to Hybrid Multi-Controller Architectures. Taylor \& Francis CRC Press, 2016.

[3] K. Bengler, K. Dietmayer, B. Farber, M. Maurer, C. Stiller, and H. Winner, "Three decades of driver assistance systems: Review and future perspectives," IEEE Intelligent Transportation Systems Magazine, vol. 6, no. 4, pp. 6-22, 2014.

[4] S. E. Lee, E. C. Olsen, W. W. Wierwille, et al., "A comprehensive examination of naturalistic lane-changes," tech. rep., United States. National Highway Traffic Safety Administration, 2004.

[5] A. Eskandarian, Handbook of intelligent vehicles, vol. 2. Springer, 2012.

[6] D. Iberraken, L. Adouane, and D. Denis, "Safe autonomous overtaking maneuver based on inter-vehicular distance prediction and multi-level bayesian decision-making," in 2018 21st International Conference on Intelligent Transportation Systems (ITSC), pp. 3259-3265, Nov 2018.
[7] T.-C. Au, C.-L. Fok, S. Vishwanath, C. Julien, and P. Stone, "Evasion planning for autonomous vehicles at intersections," in Intelligent Robots and Systems (IROS), 2012 IEEE/RSJ International Conference on, pp. 1541-1546, IEEE, 2012.

[8] B. Kim, K. Park, and K. Yi, "Probabilistic threat assessment with environment description and rule-based multi-traffic prediction for integrated risk management system," IEEE Intelligent Transportation Systems Magazine, vol. 9, no. 3, pp. 8-22, 2017.

[9] R. Schubert, "Evaluating the utility of driving: Toward automated decision making under uncertainty," IEEE Transactions on Intelligent Transportation Systems, vol. 13, no. 1, pp. 354-364, 2012.

[10] J. Hou, G. F. List, and X. Guo, "New algorithms for computing the time-to-collision in freeway traffic simulation models," Computational intelligence and neuroscience, vol. 2014, p. 57, 2014.

[11] J. R. Ward, G. Agamennoni, S. Worrall, A. Bender, and E. Nebot, "Extending time to collision for probabilistic reasoning in general traffic scenarios," Transportation Research Part C: Emerging Technologies, vol. 51, pp. 66-82, 2015.

[12] J. Hillenbrand, K. Kroschel, and V. Schmid, "Situation assessment algorithm for a collision prevention assistant," in Intelligent Vehicles Symposium, 2005. Proceedings. IEEE, pp. 459-465, IEEE, 2005.

[13] N. M. B. Lakhal, L. Adouane, O. Nasri, and J. B. H. Slama, "Risk management for intelligent vehicles based on interval analysis of ttc," in IFAC Symposium on Intelligent Autonomous Vehicles, Gdansk, Poland, pp. 1-6, July 2019.

[14] S. Mitsch, S. M. Loos, and A. Platzer, "Towards formal verification of freeway traffic control," in Proceedings of the 2012 IEEE/ACM Third International Conference on Cyber-Physical Systems, pp. 171-180, IEEE Computer Society, 2012.

[15] M. Althoff and J. M. Dolan, "Online verification of automated road vehicles using reachability analysis," IEEE Transactions on Robotics, vol. 30, no. 4, pp. 903-918, 2014.

[16] M. Althoff, D. Althoff, D. Wollherr, and M. Buss, "Safety verification of autonomous vehicles for coordinated evasive maneuvers," in Intelligent vehicles symposium (IV), 2010 IEEE, pp. 1078-1083, IEEE, 2010.

[17] M. Hilscher, S. Linker, and E.-R. Olderog, "Proving safety of traffic manoeuvres on country roads," in Theories of Programming and Formal Methods, pp. 196-212, Springer, 2013.

[18] B. $\mathrm{Xu}$ and Q. $\mathrm{Li}$, "A spatial logic for modeling and verification of collision-free control of vehicles," in Engineering of Complex Computer Systems (ICECCS), 201621 st International Conference on, pp. 33-42, IEEE, 2016.

[19] T. D. Nielsen and F. V. Jensen, Bayesian networks and decision graphs. Springer Science \& Business Media, 2009.

[20] S. Shalev-Shwartz and A. Shashua, "On the sample complexity of end-to-end training vs. semantic abstraction training," arXiv preprint arXiv:1604.06915, 2016.

[21] S. E. Lee, E. C. Olsen, W. W. Wierwille, et al., "A comprehensive examination of naturalistic lane-changes," tech. rep., United States. National Highway Traffic Safety Administration, 2004.

[22] S. J. Russell and P. Norvig, Artificial intelligence: a modern approach. Malaysia; Pearson Education Limited,, 2016.

[23] C. G. Keller, T. Dang, H. Fritz, A. Joos, C. Rabe, and D. M. Gavrila, "Active pedestrian safety by automatic braking and evasive steering," IEEE Transactions on Intelligent Transportation Systems, vol. 12, no. 4, pp. 1292-1304, 2011.

[24] M. Brannstrom, E. Coelingh, and J. Sjoberg, "Model-based threat assessment for avoiding arbitrary vehicle collisions," IEEE Transactions on Intelligent Transportation Systems, vol. 11, no. 3, pp. 658-669, 2010.

[25] A. Berthelot, A. Tamke, T. Dang, and G. Breuel, "Handling uncertainties in criticality assessment," in Intelligent Vehicles Symposium (IV), 2011 IEEE, pp. 571-576, IEEE, 2011.

[26] F. Sandblom and M. Brännström, "Probabilistic threat assessment and driver modeling in collision avoidance systems," in Intelligent Vehicles Symposium (IV), 2011 IEEE, pp. 914-919, IEEE, 2011.

[27] A. Kasmi, D. Denis, R. Aufrere, and R. Chapuis, "Map matching and lanes number estimation with openstreetmap," in 2018 21st International Conference on Intelligent Transportation Systems (ITSC), pp. 2659-2664, Nov 2018. 